Artigo Original

Original Article

\title{
Efeitos de diferentes modelos de periodização em treinamento de força sobre capacidades físicas e motoras durante 24 semanas de treinamento
}

\section{Effects of Different Periodization Models in Strength Training on Physical and Motor Skills during 24 Weeks of Training}

\begin{abstract}
Déborah de Araújo Faria ${ }^{1,2,3,4,6} \mathrm{PhD}$; Michel Moraes Gonçalves ${ }^{1,2,5} \mathrm{MSc}$; Sérgio Eduardo Nassar ${ }^{3} \mathrm{PhD}$; Euzébio de Oliveira ${ }^{3} \mathrm{PhD}$
\end{abstract}

Recebido em: 26 de setembro de 2020. Aceito em: 26 de março de 2021. Publicado online em: 29 de junho de 2021. DOI: $10.37310 /$ ref.v90i1.2693

\section{Resumo}

Introdução: Periodização é a manipulação adequada das variáveis metodológicas do treinamento de força (TF), para proporcionar o aumento progressivo das diferentes manifestações de força muscular. Os modelos mais utilizados no TF são a periodização linear e a ondulatória.

Objetivo: Avaliar os efeitos de 24 semanas de treinamento aplicando três modelos distintos de Periodização em TF: Linear (PL), Ondulatória Semanal (POS) e Ondulatória Diária (POD) sobre: força (submáxima e de resistência) de membros superiores (MMSS), força submáxima e potência de membros inferiores (MMII) e sobre outros componentes da aptidão física (flexibilidade, agilidade e força de resistência abdominal).

Métodos: Estudo experimental, longitudinal, com amostra por conveniência, do qual participaram 29 pessoas de ambos os sexos, alocados aleatoriamente nos grupos. Os testes foram realizados pré e pós-intervenção. Realizou-se ANOVA (two-way) de medidas repetidas.

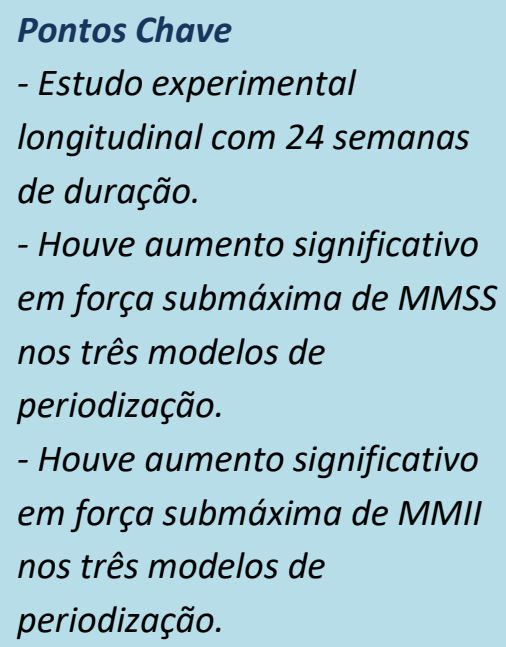

Resultados: Houve aumento significativo em força submáxima de MMSS nos três modelos de periodização: PL $(p<0,001)$, a POS $(p=0,002)$ e $\operatorname{POD}(p=0,001)$. Houve, também, aumento significativo em força submáxima de MMII com PL $(p=0,002)$, POS $(p<0,001) \mathrm{e}$ com POD $(p=0,001)$. Não foram encontradas diferenças significativas intergrupos em nenhum teste e momento.

Conclusão: Em indivíduos sem experiência em treinamento, 24 semanas de TF proporcionaram ganhos em diferentes manifestações de força, independente do modelo de periodização (PL, POS ou POD). A PL e a POS parecem ser melhores para proporcionar ganhos em potência de MMII no salto horizontal.

Palavras-chave: treinamento resistido; musculação; métodos de treinamento físico; planejamento de treinamento físico.

\footnotetext{
$\S$ Autor correspondente: Michel Moraes Gonçalves - e-mail: michel_fitness@hotmail.com Afiliações: ${ }^{1}$ Escola de Educação Física e Desportos, Universidade Federal do Rio de Janeiro - UFRJ, Rio de Janeiro, RJ, Brasil; ${ }^{2}$ LADTEF-Laboratório de Desempenho, Treinamento e Exercício Físico, Universidade Federal do Rio de Janeiro-UFRJ, Rio de Janeiro, Brasil; ${ }^{3}$ Faculdade de Educação Física, Universidade Federal do Pará - UFPA, Castanhal, PA, Brasil; ${ }^{4}$ Laboratório de Estudo do Desempenho Humano - LEDEHU, Universidade Federal do Amazonas - UFAM, Manaus, AM, Brasil; ${ }^{5}$ Escola de Educação Física do Exército - EsEFEx, Rio de Janeiro, RJ, Brasil; ${ }^{6}$ Faculdade de Educação Física, Centro Universitário do Norte - UNINORTE, Manaus, AM, Brasil.
} 


\begin{abstract}
Introduction: Periodization is the accurate manipulation of methodological variables of strength training (ST) to provide a progressive increase in the different manifestations of muscle strength. The most used models in ST are linear and undulatory periodization.

Objective: Evaluate the effects of 24 weeks of training by applying three different models of ST periodization: Linear Periodization (LP), Weekly Undulating Periodization (WUP) and Daily Undulating Periodization (DUP) on: upper limb (UL) strength (submaximal and endurance), submaximal strength and power of the lower limbs (LL) and on other components of physical fitness (flexibility, agility and abdominal endurance strength).

Methods: Experimental, longitudinal study, with a convenience sample, in which 29 people of both sexes participated, randomly allocated to the groups. Tests were performed pre- and post-intervention. ANOVA (two-way) of

\begin{tabular}{|l|}
\hline Key Points \\
- Longitudinal experimental \\
study lasting 24 weeks. \\
- There was a significant \\
increase in upper limb \\
submaximal force in the three \\
periodization models. \\
- There was a significant \\
increase in submaximal \\
strength of the lower limbs in \\
the three periodization models. \\
\hline
\end{tabular}
repeated measures was performed.

Results: There was a significant increase in submaximal strength of the UL in the three periodization models: LP $(p<0.001)$, the WUP $(p=0.002)$ and DUP $(p=0.001)$. There was also a significant increase in submaximal strength of the LL with LP $(p=0.002)$, WUP $(p<0.001)$ and with DUP $(p=0.001)$. No significant intergroup differences were found in any test and time.

Conclusion: In individuals without training experience, 24 weeks of TF provided gains in different manifestations of strength, regardless of the periodization model (LP, WUP or DUP). PL and WUP seem to be better at providing LL power gains in the horizontal jump.
\end{abstract}

Keywords: strength training; bodybuilding; physical training methods; physical training planning.

\title{
Efeitos de diferentes modelos de periodização em treinamento de força sobre capacidades físicas e motoras durante 24 semanas de treinamento
}

\section{Introdução}

A manipulação adequada das variáveis metodológicas no consideradas no planejamento do treinamento de força (TF) proporciona o aumento progressivo das diferentes manifestações de força muscular (força máxima, potência, hipertrofia, e resistência) (1). A periodização do treinamento, parte integrante do planejamento, tem por objetivo projetar ações e realizar ajustes específicos com vistas à otimização do desempenho físico e à prevenção do excesso de treinamento (2). Em estudos prévios referentes à periodização, observa-se que os modelos mais investigados são o linear (ou tradicional) e o modelo de periodização não linear (ou ondulatório) (3). O primeiro caracteriza-se por constantes incrementos da carga de treinamento com concomitante redução do volume, dispostos ao longo dos ciclos de treinamento (4). O modelo ondulatório caracteriza-se por preconizar alterações frequentes no volume $\mathrm{e}$ intensidade de treinamento, sejam estas semanais, por ciclos ou até mesmo diárias (4). Observa-se uma vasta gama de estudos referentes à temática de periodização e comparação entre seus diferentes modelos (5-8), sendo que o período mais frequentemente de aplicação da intervenção de até 16 semanas de treinamento. Outro fator observado nos estudos sobre periodização é que as variáveis mais analisadas são força máxima e submáxima (6-8), ressaltando que, na prática, tanto em treinamento físico quanto em desempenho 
esportivo, ações musculares que exijam o emprego de força máxima não é muito frequente. Tal observação indica que a funcionalidade muscular está mais relacionada com a força submáxima (9) o que ressalta a relevância de se estudar a aplicação de diferentes métodos de treinamento no desenvolvimento de força. Adicionalmente, o TF pode contribuir para aprimorar outras valências componentes da aptidão física como: velocidade, agilidade, equilíbrio, coordenação, potência e flexibilidade, além de melhorar o desempenho motor (10). Observar uma lacuna na literatura quanto à utilização de diferentes modelos de periodização sobre diferentes capacidades físicas em períodos maiores que três meses, o que justifica a relevância de se estudar o tema. Um estudo experimental utilizando um período de 24 semanas de intervenção, demonstrou que um protocolo de periodização do tipo ondulatória diária promove maiores ganhos em força de membros inferiores, enquanto para favorecer o aumento de potência de membros inferiores, a periodização do tipo ondulatória semanal mostrou-se mais eficiente (11) .

O presente estudo examinou os efeitos de 24 semanas de treinamento aplicando três modelos distintos de periodização em TF: periodização linear, periodização ondulatória semanal e periodização ondulatória diária sobre força submáxima e força de resistência de membros superiores (MMSS) e sobre força submáxima e potência de membros inferiores (MMII) e explorar os efeitos sobre outros componentes da aptidão física (flexibilidade, agilidade e força de resistência abdominal).

\section{Métodos}

\section{Desenho de estudo e amostra}

$\mathrm{O}$ presente estudo foi do tipo experimental longitudinal, com período de intervenção de 24 semanas. A amostra foi por conveniência, com alunos de graduação do curso de Educação Física da Universidade Federal do Pará (UFPA). Os participantes foram distribuídos aleatoriamente nos três grupos de

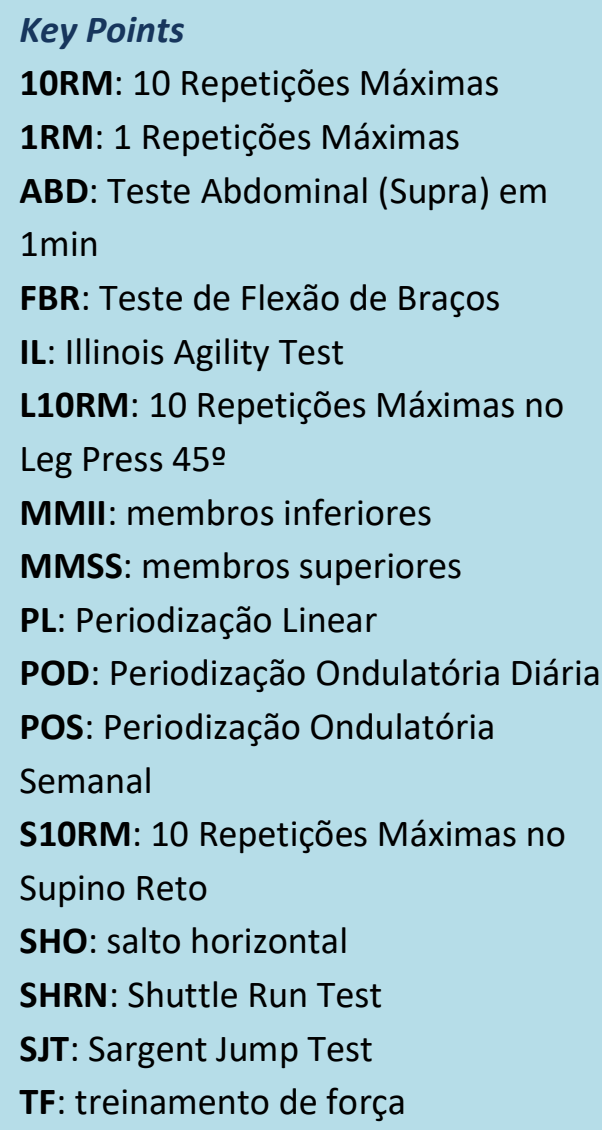

intervenção: a) Periodização Linear (PL); Periodização Ondulatória Semanal (POS); e c) Periodização Ondulatória Diária (POD).

Os critérios de inclusão foram: não ter experiência com TF e não apresentar histórico de lesões osteomioarticulares. Os critérios de exclusão foram: possuir limitação funcional para a realização dos exercícios propostos; possuir qualquer condição médica que pudesse impedir a realização das condições experimentais. Além desses, foi considerada perda amostral os indivíduos que tivessem mais de $25 \%$ de ausência no decorrer do treinamento e que faltassem três sessões seguidas de treinamento no decorrer das 24 semanas. Caso houvesse alguma ausência em alguma das sessões, a sessão de treinamento era reposta às quartas-feiras ou aos sábados.

\section{Aspectos éticos}

O projeto foi submetido ao comitê de ética em Pesquisa sob o protocolo CAAE 70890717.3.0000.0018, 
resolução 466/2012 do Conselho Nacional de Saúde para pesquisa com seres humanos.

\section{Variáveis de estudo}

As variáveis dependentes principais foram: força submáxima de membros superiores (MMSS) e de membros inferiores (MMII), força de resistência de MMSS e potência de MMII. As variáveis dependentes secundárias foram: força de resistência abdominal, flexibilidade e agilidade. A variável independente foi a intervenção experimental, composta por três modelos de periodização do treinamento de força: PL, POS e POD. Idade, massa corporal, estatura foram as covariáveis utilizadas para caracterização da amostra.

Força submáxima de membros superiores (MMSS) e membros inferiores (MMII)

Para se avaliar a força submáxima utilizou-se o Teste de 10 Repetições Máximas (RM) (12): no Supino Reto (S10RM) para MMSS e no Leg Press $45^{\circ}$ (L10RM) para MMII, descritos em detalhes em procedimento experimental.

\section{Força de resistência de membros superiores (MMSS)}

Para se avaliar a força de resistência de MMSS utilizou-se o Teste de Flexão de Braços (FBR) $(15,16)$. O participante deveria se posicionar em decúbito ventral, com as mãos apoiadas no solo, com uma distância de 10 a $20 \mathrm{~cm}$ a partir da linha dos ombros, com os dedos voltados para frente. $\mathrm{O}$ posicionamento das mãos sobre o solo não deve ser acima da linha dos ombros e, na posição inicial do movimento, o rosto deve permitir um alinhamento adequado entre o tronco e as pernas (13). A aplicação do teste para o sexo feminino é modificada apenas pelo apoio dos joelhos sobre o solo. Os demais procedimentos são realizados para ambos os sexos. Registra-se o número máximo de repetições corretas em um minuto (14).

\section{Potência de membros inferiores (MMII)}

Para avaliar a potência de MMII, foram utilizados dois testes: deslocamento vertical e deslocamento horizontal. Para avaliar a potência de MMII em deslocamento vertical utilizou-se o Sargent Jump Test (SJT) (15). Os dedos da mão direita do executante são marcados com giz, para a marcação inicial do teste. O participante realiza a extensão do braço direito acima da cabeça, lateralmente à parede e os pés totalmente apoiados no chão, marcando na parede o ponto mais alto que pôde ser alcançado. Depois da marcação inicial, o executante realiza um salto vertical, durante o qual é permitido flexionar livremente MMII e MMSS, de forma a proporcionar o maior impulso vertical possível, indicando assim, o ponto final do salto. A medida da altura máxima alcançada com a execução do salto se dá pela diferença entre os dois pontos marcados na parede, sendo realizadas três tentativas de salto, com intervalo de recuperação de 45 segundos entre as tentativas. De acordo com a metodologia do teste, considera-se como valor final, a maior marca obtida dentre as três tentativas (15).

Para avaliar a potência de MMII em deslocamento horizontal utilizou-se o teste do salto horizontal (SHO) (16). No teste, o salto horizontal é realizado partindo-se de uma linha na posição ortostática e com os pés paralelos, aproximadamente na largura dos ombros. Ao comando de "iniciar", o executante realiza o salto flexionando os joelhos e balançando os braços, para tentar obter o máximo de impulso à frente. A distância foi medida com uma trena de fibra de vidro de $25 \mathrm{~m}$, a partir da linha inicial até o ponto do calcanhar mais próximo. De acordo com a metodologia do teste, realizam-se três tentativas, sendo considerado a maior marca obtida (16).

\section{Flexibilidade}

Para se avaliar a flexibilidade foi utilizado o teste Sentar e Alcançar(17), seguindo a padronização canadense para os testes de avaliação da aptidão física do Canadian Standardized Test of Fitness (18). O teste é realizado numa caixa medindo $30,5 \mathrm{~cm} \mathrm{x}$ $30,5 \mathrm{~cm} \times 30,5 \mathrm{~cm}$ com uma escala de 26,0 $\mathrm{cm}$ em seu prolongamento, sendo que o ponto zero se encontra na extremidade mais próxima do participante e o vigésimo sexto centímetro coincide com o ponto de apoio dos pés. Os participantes realizaram os 
testes descalços e, na posição sentada, deveriam encostar os pés na caixa com os joelhos estendidos. Com ombros flexionados, cotovelos estendidos e mãos sobrepostas deve ser executada a flexão do tronco à frente devendo o participante tocar o ponto máximo da escala com as mãos. Foram realizadas três tentativas, sendo considerada apenas a melhor marca.

\section{Força de Resistência abdominal}

Para avaliar a força de resistência abdominal utilizou-se o Teste Abdominal (Supra) em 1min (ABD) (17). O participante posiciona-se em decúbito dorsal com os joelhos flexionados a 90 graus e com os braços cruzados sobre o tórax. $\mathrm{O}$ avaliador fixa os pés do participante ao solo. Ao sinal o participante inicia os movimentos de flexão do tronco até tocar com os cotovelos nas coxas, retornando à posição inicial (não é necessário tocar com a cabeça no colchonete a cada execução). O avaliador realiza a contagem em voz alta. O participante deve realizar o maior número de repetições completas em um minuto. $\mathrm{O}$ resultado é expresso pelo número de movimentos completos realizados em um minuto (18).

\section{Agilidade}

Para avaliar a habilidade motora agilidade, foram utilizados dois testes: o Shuttle Run Test (mudança abrupta de direção em $\left.180^{\circ}\right)(19)$ e Illinois Agility Test (corrida com mudança de direção e posição)(20). O Shuttle Run Test (SHRN) preconiza realizar mudança abrupta de direção em $180^{\circ}$. O teste consta de duas linhas paralelas traçadas no solo, com distância de 9,14 metros entre elas. Dois cones são colocados a 10 centímetros da linha externa e separados entre si por um espaço de 30 centímetros. Ao sinal de um comando o participante em ação simultânea, corre à máxima velocidade até os dois cones dispostos equidistantes da linha de saída à 9,14 metros. Lá chegando, pega um deles e retorna ao ponto de partida, depositando esse cone atrás da linha. Em seguida, sem interromper a corrida, vai à busca do segundo cone, procedendo da mesma forma. O cronômetro, no qual é mensurado o tempo de movimento, é parado após o participante colocar o segundo cone atrás da linha inicial. São realizadas três tentativas, com intervalo de um minuto entre as mesmas, sendo escolhida aquela com o tempo mais satisfatório (19).

O Illinois Agility Test (IL) preconiza corrida com mudança de posição e direção. O teste é configurado com quatro cones que formam a área de agilidade, 10 metros de comprimento, 5 metros de largura. Quatro cones são posicionados em cada canto da área do teste e quatro cones no centro da área de teste a 3,3 metros de distância entre eles. O participante inicia o teste deitado no solo, em decúbito ventral com as mãos ao nível do ombro e cotovelos flexionados, ao lado do cone de partida. Ao sinal do apito, o participante levanta-se e corre o percurso no caminho definido no menor tempo possível. O teste é finalizado quando o participante cruza a linha de chegada e quando nenhum dos cones são derrubados (20).

\section{Medidas Antropométricas}

Para caracterização da amostra foram tomadas as medidas antropométricas estatura e massa corporal.

\section{Intervenção}

A variável independente foi a intervenção que foi composta por três modelos distintos de periodização propostos para o TF: PL, POS e POD.

Foram realizadas quatro sessões semanais, sendo que o treinamento foi parcelado, sendo uma prescrição de treinamento para MMSS e uma prescrição de treinamento para MMII. As sessões de treinamento tiveram uma duração média de 45 minutos. A estruturação da intervenção experimental no TF segundo os modelos de periodização apresenta-se no Quadro 1.

\section{Procedimento experimental}

Os participantes foram divididos aletoriamente em três grupos sendo: grupo PL $(n=7)$, grupo POS $(n=6)$ e grupo POD $(\mathrm{n}=6)$. Foram realizadas 6 visitas ao laboratório antes do início das 24 semanas de treinamento sendo destinadas à 


\begin{tabular}{|c|c|c|c|c|}
\hline \multicolumn{5}{|c|}{ PERIODIZAÇÃO LINEAR (PL) } \\
\hline SEMANAS & $\begin{array}{c}\text { Segunda-feira } \\
\text { PARC. A MMSS }\end{array}$ & Quarta-feira & $\begin{array}{c}\text { Quinta-feira } \\
\text { PARC. A MMSS }\end{array}$ & $\begin{array}{c}\text { Sexta-feira } \\
\text { PARC. B MMII }\end{array}$ \\
\hline PRÉ & & TESTES & & \\
\hline $2^{a} a 7^{a}$ & $3 \times 12-15 R M$ & Descanso & \multicolumn{2}{|c|}{$3 \times 12-15 R M$} \\
\hline $8^{\mathbf{a}}$ & & TESTES & & \\
\hline $9^{a}$ a $15^{a}$ & $4 \times 4-5 R M$ & Descanso & \multicolumn{2}{|c|}{$4 \times 4-5 R M$} \\
\hline $16^{\mathrm{a}}$ & & TESTES & & \\
\hline $17^{\mathrm{a}}$ a $23^{\mathrm{a}}$ & $3 \times 8-10 \mathrm{RM}$ & Descanso & \multicolumn{2}{|c|}{$3 \times 8-10 \mathrm{RM}$} \\
\hline $24^{\mathrm{a}}$ & & TESTES & & \\
\hline \multicolumn{5}{|c|}{ PERIODIZAÇÃO ONDULATÓRIA SEMANAL (POS) } \\
\hline SEMANAS & $\begin{array}{cc}\text { Segunda-feira } & \text { Terça-feira } \\
\text { PARC. A MMSS } & \text { PARC. B MMII }\end{array}$ & Quarta-feira & $\begin{array}{l}\text { Quinta-feira } \\
\text { PARC. A MMSS }\end{array}$ & $\begin{array}{c}\text { Sexta-feira } \\
\text { PARC. B MMII }\end{array}$ \\
\hline $1^{\mathrm{a}}, 4^{\mathrm{a}}, 7^{\mathrm{a}}, 11^{\mathrm{a}}, 14^{\mathrm{a}}, 18^{\mathrm{a}}, 21^{\mathrm{a}}$ & $3 \times 12-15 \mathrm{RM}$ & Descanso & \multicolumn{2}{|c|}{$3 \times 12-15 R M$} \\
\hline $2^{a}, 5^{a}, 9^{a}, 12^{a}, 15^{a}, 19^{a}, 22^{a}$ & 4 x 4-5RM & Descanso & \multicolumn{2}{|c|}{$4 \times 4-5 R M$} \\
\hline $3^{a}, 6^{a}, 10^{a}, 13^{a}, 17^{a}, 20^{a}, 23^{a}$ & $3 \times 8-10 \mathrm{RM}$ & Descanso & \multicolumn{2}{|c|}{$3 \times 8-10 \mathrm{RM}$} \\
\hline PRÉ, $8^{\mathrm{a}}, 16^{\mathrm{a}}, 24^{\mathrm{a}}$ & & TESTES & & \\
\hline \multicolumn{5}{|c|}{ PERIODIZAÇÃO ONDULATÓRIA DIÁRIA (POD) } \\
\hline SEMANAS & $\begin{array}{cc}\text { Segunda-feira } & \text { Terça-feira } \\
\text { PARC. A MMSS } & \text { PARC. B MMII }\end{array}$ & Quarta-feira & $\begin{array}{l}\text { Quinta-feira } \\
\text { PARC. A MMSS }\end{array}$ & $\begin{array}{c}\text { Sexta-feira } \\
\text { PARC. B MMII }\end{array}$ \\
\hline $1^{\mathrm{a}}, 4^{\mathrm{a}}, 7^{\mathrm{a}}, 11^{\mathrm{a}}, 14^{\mathrm{a}}, 18^{\mathrm{a}}, 21^{\mathrm{a}}$ & $3 \times 12-15 \mathrm{RM}$ & Descanso & \multicolumn{2}{|c|}{$3 \times 8-10 \mathrm{RM}$} \\
\hline $2^{a}, 5^{a}, 9^{a}, 12^{a}, 15^{a}, 19^{a}, 22^{a}$ & 4 x 4-5RM & Descanso & \multicolumn{2}{|c|}{$3 \times 12-15 R M$} \\
\hline $3^{\mathrm{a}}, 6^{\mathrm{a}}, 10^{\mathrm{a}}, 13^{\mathrm{a}}, 17^{\mathrm{a}}, 20^{\mathrm{a}}, 23^{\mathrm{a}}$ & $3 \times 8-10 \mathrm{RM}$ & Descanso & \multicolumn{2}{|c|}{4 x 4-5RM } \\
\hline PRÉ, $8^{\mathrm{a}}, 16^{\mathrm{a}}, 24^{\mathrm{a}}$ & & TESTES & & \\
\hline
\end{tabular}

Quadro 1 - Intervenção experimental em treinamento de força (TF) segundo modelos de periodização: periodização linear (PL), periodização ondulatória semanal (POS) e periodização ondulatória diária (POD)

PARC:: parcelamento; MMSS: membros superiores; MMII: membros inferiores; RM: repetições máximas. 
familiarização tanto com os exercícios como com os testes de 10 repetições máximas. $\mathrm{O}$ Quadro 2 apresenta o fluxograma do protocolo experimental.

Durante a primeira visita ao laboratório foi feita uma explanação do procedimento experimental e assinatura do TCLE.

Quarenta e oito horas após a última sessão de familiarização, foi realizada a primeira visita da semana de testes para mensurar a massa corporal $(\mathrm{kg})$ e a estatura $(\mathrm{m})$. A mensuração da massa corporal $(\mathrm{kg})$ foi realizada em uma balança digital de marca Toledo 2096 PP (São Bernardo do Campo, $\mathrm{SP}$, Brasil) enquanto a altura $(\mathrm{cm})$ foi realizada em um estadiômetro da marca Wiso (Florianópolis, SC, Brasil). Estas variáveis foram medidas apenas no período pré-experimental para caracterização do grupo, bem como foram aplicados os testes de Sentar e alcançar (flexibilidade), Sargent Jump Test e Salto horizontal (potência de MMII), Flexão de braços (força de resistência de MMSS), Teste de abdominal supra 1 min (força de resistência abdominal), Shuttle Run e Illinois Agility Test (agilidade).

$\mathrm{Na}$ segunda visita foram realizados os testes de 10 repetições máximas (10RM), Supino reto e Leg Press $45^{\circ}$. Após a sessão de familiarização com os exercícios propostos (supino reto e leg press $45^{\circ}$ ), todos os sujeitos realizaram uma sessão de familiarização com o protocolo do teste de 10RM com 48 horas de intervalo entre as sessões. Em um dia foi executado o primeiro teste de 10RM, após 48 horas de intervalo, um segundo teste de 10RM ocorreu para verificar se houve reprodutibilidade entre teste-reteste. A maior carga alcançada entre os dois dias foi considerada a 10RM pré-treinamento. Os sujeitos não realizaram nenhum exercício no intervalo entre os dois dias de teste. $\mathrm{O}$ teste de 10RM teve no máximo cinco tentativas com cinco minutos de intervalo entre as mesmas e 10 minutos entre os exercícios. Caso um dos exercícios apresentasse necessidade da sexta tentativa, o mesmo foi testado novamente em outro dia após 48 horas de intervalo (21).
$\mathrm{Na}$ terceira visita foram realizados os retestes de 10RM.

Entre as visitas, houve um intervalo de recuperação de 48 horas. O intervalo de 48 horas também foi aplicado entre o reteste de 10RM e o início da sessão de treinamento.

A cada oito semanas esses testes foram reaplicados para avaliar a aptidão física e reajustar a intensidade do treinamento dos participantes. Desta forma, as avaliações foram realizadas antes do início das sessões de treinamento (pré), na semana 8 , na semana 16, e ao final das 24 semanas (pós), totalizando assim quatro avaliações no decorrer das 24 semanas (11) (Quadro 1).

\section{Sessões de Treinamento}

Após o intervalo de 48 a 72 horas do reteste de 10RM foi dado início às sessões de treinamento. Cada participante foi alocado aleatoriamente em um dos grupos experimentais (PL, POS e POD) e realizou um total de 96 sessões no decorrer das 24 semanas. A duração do intervalo de recuperação (IR) entre séries e exercícios utilizados foram de acordo com a recomendação do American College of Sports Medicine (22), ou seja: para Resistência Muscular Localizada (RML) sessenta segundos entre as séries e exercícios, para hipertrofia muscular 90 segundos, e para força muscular foi dado um IR de três minutos. Os exercícios para membros superiores, utilizados no parcelamento $\mathrm{A}$, foram supino reto com barra, voador, tríceps na polia, puxada pela frente, remada sentado e rosca direta na polia. Os exercícios para membros inferiores, utilizados no parcelamento B, foram mesa flexora, agachamento no Smith Machine, Leg Press $45^{\circ}$, flexão plantar na máquina em pé e abdominal.

\section{Análise estatística}

Os valores foram expressos em média e desvio padrão. A análise da normalidade na distribuição dos dados foi feita a partir do teste de Shapiro-Wilk. Foi aplicada uma ANOVA (one-way) para analisar a diferença significativa entre os grupos no 


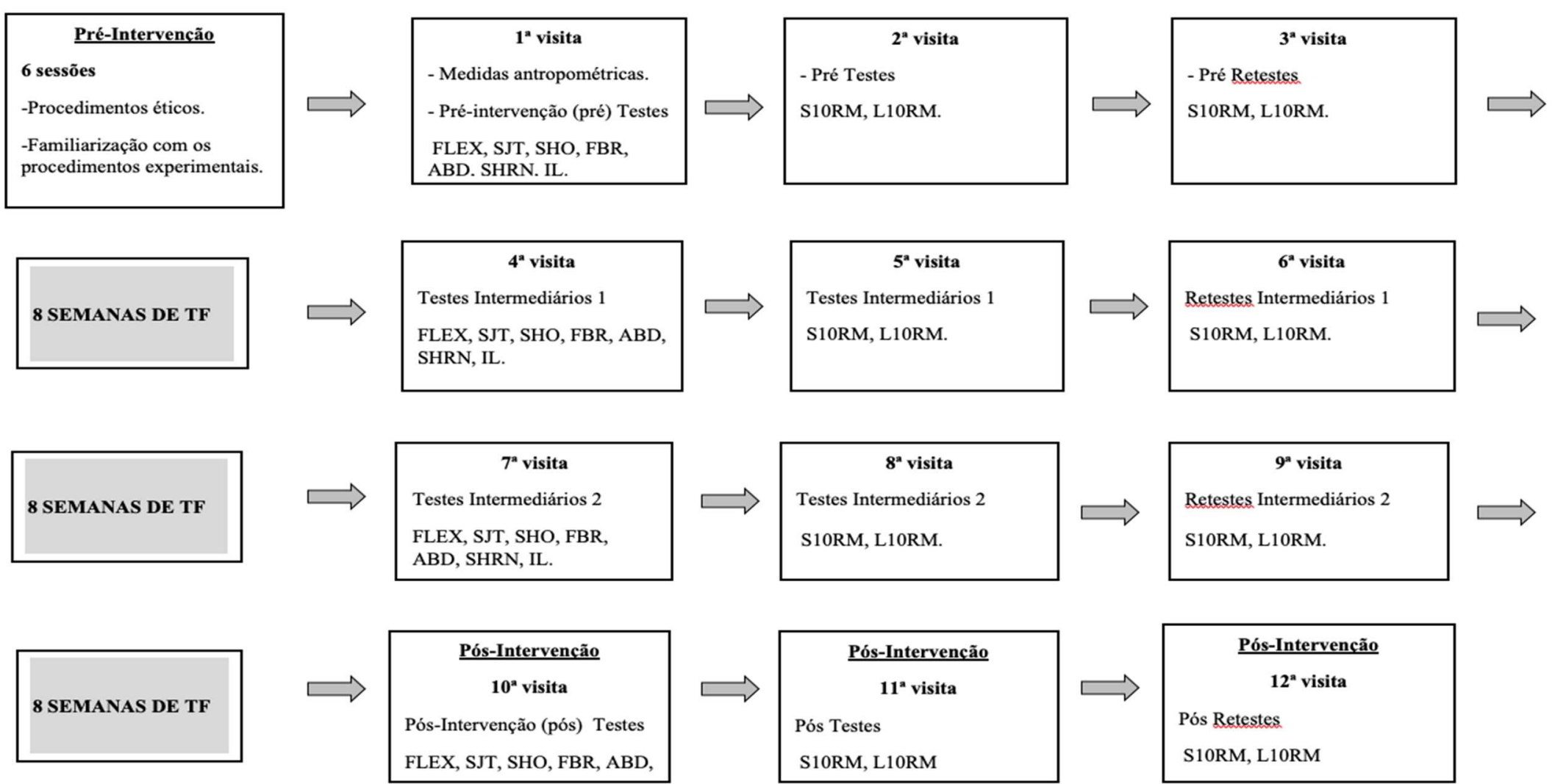

Quadro 2 - Fluxograma do protocolo experimental para comparação dos efeitos de três métodos distintos de periodização em treinamento de força (TF): Periodização Linear (PL); Periodização Ondulatória Semanal (POS); e Periodização Ondulatória Diária (POD)

FLEX: teste de sentar e alcançar (flexibilidade); SJT: Sargent Jump Test (potência de membros inferiores (MMII) em deslocamento vertical); SHO: salto horizontal (potência de MMII em deslocamento horizontal); FBR: teste de flexão de braços (força de resistência de membros superiores (MMSS)); ABD: Teste de abdominal de 1 minuto (força de resistência abdominal); SHRN: Shuttle Run (agilidade: direção); IL: Illinois Agility Run (agilidade: direção em deslocamento); S10RM: teste de 10 repetições em carga máxima no supino reto (força submáxima de MMSS); L10RM: 10 repetições máximas no Leg press $45^{\circ}$ (força submáxima de membros inferiores (MMII)). 
momento Pré e a ANOVA (two-way) de medidas repetidas com pos hoc de Bonferroni foi aplicada para analisar as diferenças entre os diferentes momentos de testes (Pré, semana 8, semana 16 e Pós) nos diferentes modelos de periodização. $\mathrm{O}$ valor alfa utilizado para todas as etapas de análises experimentais foi de $p \leq 0,05$. A versão 22.0 do SPSS software for Mac (SPSS Inc., Chicago, IL, USA) foi aplicada em todas as análises estatísticas.

\section{Resultados}

Dos 45 convidados para participar do estudo houve uma perda de 26 participantes, pelo critério de exclusão de mais de $25 \%$ de ausência às sessões de treinamento. Assim, a amostra foi composta por 19 participantes $(9$ mulheres e 10 homens). As características dos voluntários estão apresentadas na Tabela 1.

Os resultados das avaliações nos diferentes momentos de testes para os três grupos apresentam-se na Tabela 2. Não foram encontradas diferenças entre os grupos no momento pré-intervenção em força submáxima de MMSS ( $\mathrm{p}=0,256)$, e MMII $(p=0,887)$, força de resistência de MMSS $(p=0,426)$, potência de MMII em deslocamento vertical $(p=0,352)$ e em deslocamento horizontal $(\mathrm{p}=0,478)$, nem em agilidade de direção $(\mathrm{p}=0,410)$ e em agilidade de direção com deslocamento $(p=0,285)$, em flexibilidade $(p=0,676)$, e nem em força de resistência abdominal $(p=0,560)$. Não foram encontradas diferenças significativas intergrupos (PL, POS e POD) em nenhum momento (PréIntervenção, Semana 8, Semana 16 e PósIntervenção), em nenhum dos testes.

A Tabela 3 apresenta os resultados de força de resistência, flexibilidade e agilidade Média dos momentos intragrupos.

Em força de resistência abdominal, no grupo PL foram observadas diferenças significativas no período pré comparado à semana $16(p=0,050)$; pré comparado ao período pós $(p=0,009)$; semana 8 se comparada ao período pós $(p=0,001)$; semana 16 se comparada ao período pós ( $p$ $=0,004)$. No grupo POS, foram observadas diferenças significativas no período pré comparado à semana $16(p=0,011)$; pré comparado ao período pós $(p=0,004)$ e semana 8 se comparada ao período pós $(p=$ $0,021)$. No grupo POD, foram observadas diferenças significativas no período pré comparado ao pós $(p=0,050)$.

Quanto à flexibilidade, houve diferença significativa apenas para o grupo POS do período pré para a semana $8(p=0,023)$.

Em agilidade, foram observadas diferenças significativas em mudança abrupta de direção (Shuttle Run Test) no grupo PL: do período pré para o período pós $(p=0,003)$ e da semana 8 para o período pós $(p=0,006)$; no grupo POS: foram observadas diferenças significativas na semana 8 para o período pós $(p=0,038)$; e no grupo POD: foram observadas diferenças significativas apenas do período pré para o pós $(p=0,020)$.

Em força submáxima de MMSS, o grupo PL apresentou diferenças significativas do período pré para a semana $8(p<0,001)$; do período pré para a semana $16(p=0,003)$; do período pré para o período pós $(p<0,001)$; da semana 8 para a semana $16(p=0,027)$; da semana 8 para o período pós $(p=0,003)$. No grupo POS, foram observadas diferenças significativas do período pré para a semana $8(p=0,003)$; do período pré para a semana 16 ( $p=0,006)$; do período pré para o período pós $(p=0,002)$; da semana 8 para o período pós $(p=0,007)$; da semana 16 para o período pós $(p=0,007)$. Para o grupo POD foram observadas diferenças significativas do período pré para a semana $8(p=0,007)$; do período pré para a semana $16(p=0,002)$; do período pré para o período pós $(p=0,001)$; da semana 8 para a semana $16(p=0,009)$; da semana 8 para o período pós $(p=0,002) \mathrm{e}$ da semana 16 para o período pós $(p=0,014)$.

Em força submáxima de MMII, o grupo PL apresentou diferenças significativas do período pré para a semana $8(p=0,014)$; do período pré para a semana $16(p=0,006)$; do período pré para o período pós $(p=0,002)$; da semana 8 para o período pós $(p=0,009)$ e da semana 16 para o período pós $(p=0,015)$. No grupo POS, foram observadas diferenças significativas do período pré 
Tabela 1 - Caracterização da amostra e distribuição dos participantes pelos grupos de intervenção: Periodização Linear (PL), Periodização Ondulatória Semanal (POS) e Periodização Ondulatória Diária (POD) $(\mathrm{n}=19)$

\begin{tabular}{|c|c|c|c|}
\hline Grupo & $\begin{array}{c}\text { Idade } \\
(\mathbf{a n o s} \pm \mathbf{D P})\end{array}$ & $\begin{array}{c}\text { Massa corporal } \\
\mathbf{( k g} \pm \mathbf{D P})\end{array}$ & $\begin{array}{c}\text { Altura } \\
(\mathbf{m} \pm \mathbf{D P})\end{array}$ \\
\hline \hline PL & $23 \pm 3,20$ & $59,70 \pm 4,20$ & $1,66 \pm 0,05$ \\
\hline POS & $24 \pm 2,10$ & $65,10 \pm 8,70$ & $1,69 \pm 0,10$ \\
\hline POD & $23 \pm 1,70$ & $65,80 \pm 3,90$ & $1,70 \pm 0,10$ \\
\hline
\end{tabular}

PL: Periodização Linear; POS: Periodização Ondulatória Semanal; POD: Periodização Ondulatória Diária; kg: quilograma; m: metro.

Tabela 2 - Força submáxima de membros superiores (MMSS) e membros inferiores (MMII) e potência de MMII em deslocamento vertical e horizontal segundo modelo de periodização de treinamento de força $(\mathrm{TF})(\mathrm{n}=19)$

\begin{tabular}{|c|c|c|c|c|}
\hline Avaliações & Momentos & $\begin{array}{l}\text { Periodização } \\
\text { Linear (PL) } \\
(n=7)\end{array}$ & $\begin{array}{c}\text { Periodização } \\
\text { Ondulatória } \\
\text { Semanal (POS) } \\
(n=6)\end{array}$ & $\begin{array}{c}\text { Periodização } \\
\text { Ondulatória } \\
\text { Diária (POD) } \\
(n=6)\end{array}$ \\
\hline \multirow{4}{*}{$\begin{array}{l}\text { Força } \\
\text { submáxima } \\
\text { MMSS }^{\mathrm{a}}\end{array}$} & Pré & $23,14 \pm 9,73$ & $29,16 \pm 14,86$ & $35,16 \pm 13,02$ \\
\hline & Semana 8 & $25,71 \pm 9,70 *$ & $36,50 \pm 17,08^{*}$ & $39,16 \pm 13,02 *$ \\
\hline & Semana 16 & $31,00 \pm 13,16^{* \&}$ & $40,16 \pm 17,90^{*}$ & $46,50 \pm 15,12 * \&$ \\
\hline & Pós & $33,00 \pm 12,75^{* \&}$ & $43,16 \pm 18,96^{* \& \mathfrak{E}}$ & $\begin{array}{c}49,50 \pm \\
13,64 * \& \mathfrak{A}\end{array}$ \\
\hline \multirow{4}{*}{$\begin{array}{l}\text { Força } \\
\text { submáxima }^{\text {MMSSII }}{ }^{b}\end{array}$} & Pré & $114,57 \pm 59,11$ & $126,66 \pm 55,73$ & $127,00 \pm 38,75$ \\
\hline & Semana 8 & $138,85 \pm 74,35^{*}$ & $144,66 \pm 57,22$ & $164,33 \pm 43,73 *$ \\
\hline & Semana 16 & $162,28 \pm 81,50 *$ & $\begin{array}{l}169,33 \pm \\
66,71^{* \&}\end{array}$ & $\begin{array}{l}200,00 \pm \\
50,99 * \&\end{array}$ \\
\hline & Pós & $\begin{array}{l}192,00 \pm \\
85,60 * \& \mathfrak{f}\end{array}$ & $\begin{array}{l}208,33 \pm \\
61,12^{* \& \mathfrak{f}}\end{array}$ & $\begin{array}{l}233,00 \pm \\
57,15^{* \& \mathfrak{x}}\end{array}$ \\
\hline \multirow{4}{*}{$\begin{array}{l}\text { Potência MMII } \\
\text { desloc. } \\
\text { Vertical }^{c}\end{array}$} & Pré & $0,34 \pm 0,07$ & $0,39 \pm 0,13$ & $0,43 \pm 0,09$ \\
\hline & Semana 8 & $0,41 \pm 0,09$ & $0,44 \pm 0,15$ & $0,44 \pm 0,13$ \\
\hline & Semana 16 & $0,39 \pm 0,05$ & $0,48 \pm 0,14$ & $0,44 \pm 0,08$ \\
\hline & Pós & $0,38 \pm 0,06$ & $0,51 \pm 0,15$ & $0,44 \pm 0,10$ \\
\hline \multirow{4}{*}{$\begin{array}{l}\text { Potência MMII } \\
\text { desloc. } \\
\text { Horizontal }^{\mathrm{d}}\end{array}$} & Pré & $1,45 \pm 0,39$ & $1,69 \pm 0,41$ & $1,66 \pm 0,34$ \\
\hline & Semana 8 & $1,54 \pm 0,35^{*}$ & $1,85 \pm 0,45^{*}$ & $1,87 \pm 0,44$ \\
\hline & Semana 16 & $1,61 \pm 0,31^{*}$ & $1,88 \pm 0,48^{*}$ & $2,01 \pm 0,52$ \\
\hline & Pós & $1,58 \pm 0,32 *$ & $1,88 \pm 0,45^{*}$ & $1,82 \pm 0,26$ \\
\hline
\end{tabular}

Teste S10RM: 10 repetições máximas no supino reto; ${ }^{\mathbf{b}}$ Teste L10RM: 10 repetições máximas no Leg press $45^{\circ}$; 'SJT: Sargent Jump test; 'SHO: salto horizontal. *Diferença significativa com relação ao período pré; \& Diferença significativa com relação à semana 8; £ Diferença significativa com relação à semana 16. 
Tabela 3 - Média e desvio padrão dos momentos intragrupos nos testes de Força de Resistência, Flexibilidade e Agilidade

\begin{tabular}{|c|c|c|c|c|}
\hline Avaliações & Momentos & $\begin{array}{c}\text { Periodização } \\
\text { Linear }(n=7)\end{array}$ & $\begin{array}{c}\text { Periodização } \\
\text { Ondulatória } \\
\text { Semanal }(n=6)\end{array}$ & $\begin{array}{c}\text { Periodização } \\
\text { Ondulatória } \\
\text { Diária }(n=6)\end{array}$ \\
\hline \multirow{4}{*}{$\begin{array}{l}\text { Força de } \\
\text { resistência } \\
\text { MMSS }^{\mathrm{a}}\end{array}$} & Pré & $19,42 \pm 10,69$ & $25,00 \pm 8,22$ & $19,00 \pm 6,22$ \\
\hline & Semana 8 & $22,00 \pm 5,62$ & $26,00 \pm 5,72$ & $26,33 \pm 8,16$ \\
\hline & Semana 16 & $23,57 \pm 4,82$ & $31,66 \pm 7,60 * \&$ & $31 \pm 6,92 *$ \\
\hline & Pós & $27,42 \pm 6,57 * \&$ & $34,16 \pm 7,62 * \& \varepsilon$ & $33,33 \pm 7,44 * \&$ \\
\hline \multirow{4}{*}{$\begin{array}{l}\text { Força de } \\
\text { resistência } \\
\text { abdominal }^{\text {b }}\end{array}$} & Pré & $24,57 \pm 12,50$ & $30,16 \pm 7,35$ & $29,33 \pm 8,91$ \\
\hline & Semana 8 & $29,85 \pm 6,93$ & $30,50 \pm 7,68$ & $34,83 \pm 11,85$ \\
\hline & Semana 16 & $31,71 \pm 12,85 *$ & $34,83 \pm 7,16^{*}$ & $34,66 \pm 6,25 *$ \\
\hline & Pós & $37,14 \pm 10,10 * \& £$ & $37,16 \pm 9,10 * \&$ & $36,83 \pm 9,47 *$ \\
\hline \multirow[t]{4}{*}{ Flexibilidade $^{c}$} & Pré & $31,28 \pm 6,73$ & $28,5 \pm 4,49$ & $28,16 \pm 8,95$ \\
\hline & Semana 8 & $30,64 \pm 5,22$ & $33,08 \pm 3,90 *$ & $30,58 \pm 9,59$ \\
\hline & Semana 16 & $31,35 \pm 6,70$ & $35,00 \pm 7,70$ & $31,75 \pm 11,27$ \\
\hline & Pós & $29,32 \pm 6,92$ & $36,00 \pm 7,56$ & $32,00 \pm 10,2$ \\
\hline \multirow{4}{*}{$\begin{array}{l}\text { Agilidade } \\
\text { (mudança } \\
\text { direção e } \\
\text { posição) }^{\text {d }}\end{array}$} & Pré & $19,01 \pm 2,32$ & $17,44 \pm 1,74$ & $17,44 \pm 2,03$ \\
\hline & Semana 8 & $19,23 \pm 1,87$ & $18,61 \pm 2,07$ & $18,12 \pm 1,59$ \\
\hline & Semana 16 & $19,17 \pm 1,10$ & $19,09 \pm 2,23$ & $18,21 \pm 1,63$ \\
\hline & Pós & $19,75 \pm 1,63$ & $18,22 \pm 2,14$ & $18,61 \pm 1,59$ \\
\hline \multirow{4}{*}{$\begin{array}{l}\text { Agilidade } \\
(\text { mudança } \\
\left.\text { direção } 180^{\circ}\right)^{\mathrm{e}}\end{array}$} & Pré & $11,22 \pm 1,37$ & $10,55 \pm 0,66$ & $10,55 \pm 0,77$ \\
\hline & Semana 8 & $11,32 \pm 1,06$ & $10,77 \pm 1,06$ & $10,15 \pm 0,89$ \\
\hline & Semana 16 & $10,90 \pm 1,02$ & $10,60 \pm 1,08$ & $10,37 \pm 0,90$ \\
\hline & Pós & $10,52 \pm 1,19 * \&$ & $10,43 \pm 1,11 * \&$ & $10,03 \pm 0,45 *$ \\
\hline
\end{tabular}

a FBR: teste de flexão de braços; ${ }^{\mathbf{b}}$ ABD - Teste de abdominal de 1 minuto; ${ }^{\mathbf{c}}$ FLX - teste de sentar e alcançar; ${ }^{\mathbf{d}}$ IL - Illinois Agility Run; ${ }^{\text {e } S H R N ~-~ S h u t t l e ~ R u n . ~ * ~ D i f e r e n c ̧ a ~ s i g n i f i c a t i v a ~ c o m ~ r e l a c ̧ a ̃ o ~ a o ~ p e r i ́ o d o ~ p r e ́ ; ~ \& ~ D i f e r e n c ̧ a ~}$ significativa com relação à semana 8; $£$ Diferença significativa com relação à semana 16 .

para a semana $16(p=0,004)$; do período pré para o período pós $(p<0,001)$; da semana 8 para a semana $16(p=0,012)$ da semana 8 para o período pós $(p=0,001)$; da semana 16 para o período pós $(p=0,002)$. Para o grupo POD foram observadas diferenças significativas do período pré para a semana $8(p=0,003)$; do período pré para a semana 16 ( $p=0,003)$; do período pré para o período pós $(p=0,001)$; da semana 8 para a semana $16(p=0,010)$; da semana 8 para o período pós $(p=0,001)$ e da semana 16 para $o$ período pós $(p=0,001)$.

Quanto à potência de MMII, em deslocamento horizontal, foram observadas diferenças significativas no grupo PL e no grupo POS do período pré para a semana 8 ( $p=0,031$; e $p=0,001$, respectivamente); do período pré para a semana $16(p=0,024$; e $p=0,023)$ e do período pré para o período pós $(p=0,046$; e $p=0,015)$. Já no grupo POD não foram observadas diferenças significativas intragrupo, ou seja, nos resultados entre os momentos. Em relação à potência de MMII, em deslocamento vertical, não se observou diferença significativa intragrupos para nenhum dos modelos de periodização.

Em força de resistência de MMSS, o grupo PL apresentou diferenças significativas do período pré para o período pós $(p=0,004)$ e da semana 8 para o período pós $(p=0,002)$. No grupo a POS, foram observadas diferenças significativas do período pré para o período pós $(p=0,039)$; da semana 8 para a semana $16(p=0,025)$ da semana 8 para o período pós $(p=0,007)$; da semana 16 para o período pós $(p=0,004)$. No grupo POD foram observadas diferenças significativas do período pré para a semana $16(p=0,005)$; do período pré 
para o período pós $(p=0,008)$ e da semana 8 para o período pós $(p=0,046)$.

\section{Discussão}

O principal achado do presente estudo foi que, em indivíduos inexperientes, após 24 semanas de TF, houve aumento significativo em força submáxima de MMSS e MMII para os três modelos de periodização que compuseram o experimento (PL, POS e POD) (Tabela 2). Estes achados corroboram estudos prévios que demonstraram que para sujeitos destreinados ou treinados recreacionalmente(23), não houve diferença em ganhos de força máxima na comparação da aplicação de diferentes modelos de periodização $(6,24)$. Outros autores encontraram resultados semelhantes mesmo em sujeitos com características diferentes como, atletas (25-27), indivíduos experientes em TF $(28,29)$ e sedentários (30).

No presente estudo, os resultados em ganhos de força submáxima, observados em todos os modelos de periodização, estão em linha com estudos em força máxima, pois, há forte correlação entre força máxima e força submáxima (31). Além disso, em sujeitos destreinados, essa relação parece ser ainda mais forte e aumentar a magnitude após um período de TF (32), sendo que, funcionalmente, é mais importante melhorar a capacidade de exercer força contra cargas leves, ou seja, aumentar a força submáxima (9).

Destaca-se que, para sujeitos com experiência em TF, estudos indicam que a periodização ondulatória parece apresentar vantagem sobre a linear em ganhos de força máxima $(7,8)$. Porém, para indivíduos sem experiência em $\mathrm{TF}$, como é o caso do presente estudo, revisões sistemáticas não haver diferenças significativas entre os modelos de periodização linear ou ondulatório, corroborando nossos resultados $(4,33)$.

Em relação aos ganhos de potência de MMII, foram observados resultados distintos em deslocamento horizontal e deslocamento vertical: não foram encontrados ganhos significativos em deslocamento vertical e houve aumento significativo em potência de MMII em deslocamento horizontal, em relação ao desempenho no pré-teste em comparação aos testes nas semanas 8,16 e pós teste, tanto na PL, quanto na POS, o que não se observou na POD (Tabela 2). Tais achados podem ser explicados pelos diferentes padrões de ativação muscular promovidos pelos dois tipos de deslocamento (saltos vertical e horizontal. Comparando-se o salto horizontal com o salto vertical, na execução do primeiro há uma exigência maior nos músculos do quadril (34), sendo que existem evidências de que o TF, mesmo não específico, pode promover ganhos de força nos músculos do quadril $(35,36)$. Assim, pode-se inferir que o TF promoveu possíveis ganhos de força no quadril o que pode ter contribuído para os ganhos significativos em potência de MMII em deslocamento horizontal, embora não explique por que o mesmo não ocorreu com o grupo que treinou com POD. Isto ocorreu, provavelmente, pela característica da amostra, que teve mais facilidade em aprender o gesto motor do salto horizontal do que do salto vertical.

A ausência de ganhos significativos em potência de MMII em deslocamento vertical com o TF, em nenhum modelo de periodização aplicado difere do estudo que comparou os resultados do TF utilizando POD e PL, em 14 bombeiros jovens $(21,9 \pm 1,8$ anos) do sexo masculino (37). Os autores concluíram que ambas as periodizações proporcionaram ganhos em potência de MMII em deslocamento vertical. O mesmo ocorreu em outro estudo, onde os autores observaram resultados semelhantes com a aplicação de modelos POS e PL em 33 homens jovens $(20,0 \pm 2,6$ anos) com experiência em TF, sendo que ambos os modelos de periodização de $\mathrm{TF}$ também proporcionaram ganhos em potência de MMII em deslocamento vertical (38). Uma possível explicação para a diferença dos resultados daqueles estudos com o nosso, está no teste de salto, devido a protocolos de salto diferentes (Counter Movement Jump e Sargent Jump Test), uma 
vez que o SJT é executado com o auxílio dos braços, diferente do CMJ.

No presente estudo, todos os modelos de periodização proporcionaram ganhos em força de resistência de MMSS e de musculatura abdominal entre os testes pré e pós, após 24 semanas de TF (Tabela 3). Porém, nos grupos que treinaram com modelos ondulatórios, os ganhos significativos na força de resistência de MMSS ocorreram com menos tempo de TF, na semana 16. Em um estudo envolvendo 60 voluntários, 30 homens e 30 mulheres, estudantes universitários com experiência em $\mathrm{TF}$, que realizaram $\mathrm{TF}$ para membros inferiores com periodização linear, linear reversa ou POD, os autores não encontraram diferença significativa nos ganhos de RML, medido pelo número de repetições de extensões de joelho em dinamômetro isocinético, em nenhum dos grupos, após 6 semanas de treinamento, porém, encontrou após 15 semanas (39). Em um estudo com 28 mulheres jovens e sedentárias, que realizaram $\mathrm{TF}$ com periodização linear ou POD, após 12 semanas de treinamento, os autores observaram que ambos os grupos tiveram ganhos de RML em testes de números de repetições em membros superiores e inferiores, e não houve diferença significativa entre os grupos (30). Uma possível explicação para as diferenças entre os resultados destes estudos com o nosso, é que em ambos os trabalhos o programa de TF foi específico para ganhos de resistência muscular, composto por séries com 15, 20 ou 25RM.

Estudos prévios indicam que o TF pode proporcionar efeitos crônicos de melhora na flexibilidade (40-42). Entretanto, neste trabalho não foi encontrada diferença significativa entre os resultados nos momentos pré e pós TF com PL, POS ou POD no teste de flexibilidade de sentar e alcançar. Somente foi encontrada diferença significativa no grupo POS entre os resultados nos momentos pré-teste e na semana 8. Porém, apesar de não haver diferença significativa, o comportamento dos resultados de flexibilidade nos grupos POS e POD foi de ganho progressivo a cada teste, pré-teste, semana 8 , semana 16 e pós teste (Tabela 3), sinalizando que há indícios de uma possível vantagem do TF com modelos de periodização ondulatória sobre o modelo linear nos ganhos de flexibilidade com o TF. Estudos que também mediram a flexibilidade após protocolos de $\mathrm{TF}$ por intermédio do teste de flexibilidade de sentar e alcançar encontraram resultados semelhantes, ou seja, sem ganhos na PL (43), e com ganhos significativos nos modelos ondulatórios (44). Os benefícios do $\mathrm{TF}$ na flexibilidade estariam relacionados a adaptações no tecido conjuntivo e amplitude de movimento articular (45). Parece haver vantagem nos modelos ondulatórios sobre o linear tradicional (46), embora os motivos ainda não estejam bem esclarecidos.

Em relação à agilidade, o TF não teve efeito em mudança de direção em deslocamento independente do modelo de periodização. Além disso, houve perda significativa no desempenho de agilidade em mudança abrupta de direção, na PL e na POD. Na PL, houve declínio entre o préteste e o pós-teste, e entre a semana 8 e pós teste, na periodização linear; entre a semana 8 e pós teste, na POS; e entre o pré-teste e o pós-teste na POD (Tabela 3). Tais achados sugerem que o TF não influenciou ou até diminuiu a agilidade, sendo estes efeitos negativos observados mais rapidamente na PL. Isso talvez tenha ocorrido porque, ao realizarem o teste na semana 8 , os voluntários estavam encerrando o mesociclo de força de resistência (endurance). A literatura indica que parece não haver relação entre a agilidade e a força de resistência de membros inferiores(47). Os achados do presente estudo estão em concordância com outros estudos que apontaram que os ganhos de força proporcionados pelo TF não parecem estar relacionados a ganhos em agilidade, independentemente do volume de treinamento (48) ou se aplicado apenas em MMII ou em uma rotina de treinamento do corpo todo (49). Apesar disso, existem evidências que o TF de longo prazo pode proporcionar ganhos no desempenho em 
agilidade em mudança abrupta de direção (50)

\section{Pontos fortes e limitações do estudo}

Um ponto forte do estudo foi o desenho de estudo: experimental, de caráter longitudinal. Isto porque a maioria dos estudos prévios identificados examinaram a periodização em TF em períodos de duração entre 8 e 16 semanas de treinamento, e neste estudo, o período de intervenção foi de 24 semanas de TF, o que imprime maior robustez aos efeitos de longo prazo do treinamento físico.

Outro ponto que ressalta a relevância do presente estudo foi que há escassez de estudos que tenham comparado programas de TF com aplicação dos três modelos de periodização: PL, POS e POD, sendo que a maioria dos estudos envolve apenas dois dos modelos. Assim, estes resultados constituem-se em importante contribuição para preencher a lacuna no conhecimento existente sobre o tema.

Apesar de ter havido uma fase de adaptação ao protocolo experimental, uma possível limitação do estudo foi a capacidade de aprendizado de cada voluntário, além da motivação individual para realizar o TF. A ordem de execução dos exercícios foi aleatória, sendo realizada de acordo com a escolha do voluntário.

\section{Conclusão}

O presente estudo teve por objetivo analisar os efeitos de diferentes modelos de periodização linear, ondulatória semanal e ondulatória diária no TF sobre a força submáxima, potência, força de resistência, flexibilidade e agilidade em acadêmicos do curso de educação física, iniciantes com a prática do TF.

Conclui-se que, para indivíduos sem experiência, 24 semanas de $\mathrm{TF}$ proporcionaram ganhos em diferentes manifestações de força, independente do modelo de periodização (PL, POS ou POD). A PL e a POS parecem ser melhores para proporcionar ganhos em potência de MMII no salto horizontal, mas não foram observados ganhos significativos no salto vertical, independentemente do modelo de periodização. Todos os modelos de periodização aplicados neste estudo proporcionaram ganhos de resistência muscular, mas, os modelos ondulatórios parecem proporcionar ganhos mais rapidamente. Realizar apenas o $\mathrm{TF}$ parece não influenciar na flexibilidade e, até mesmo, pode prejudicar a agilidade, independente do modelo de periodização adotado.

Sendo assim, acreditamos que profissionais e pesquisadores de educação física e demais áreas da saúde podem se beneficiar dos resultados deste estudo em relação à seleção do melhor modelo de periodização de $\mathrm{TF}$, de acordo com a valência física e motora a ser desenvolvida.

\section{Declaração de conflito de interesses}

Não há nenhum conflito de interesses em relação ao presente estudo.

\section{Declaração de financiamento}

O presente trabalho foi realizado com apoio da Coordenação de Aperfeiçoamento de Pessoal de Nível Superior - Brasil (CAPES) - Código de Financiamento 001.

\section{Referências}

1. Garber CE, Blissmer B, Deschenes MR, Franklin BA, Lamonte MJ, Lee I-M, et al. American College of Sports Medicine position stand. Quantity and quality of exercise for developing and maintaining cardiorespiratory, musculoskeletal, and neuromotor fitness in apparently healthy adults: guidance for prescribing exercise. Medicine and Science in Sports and Exercise. [Online] 2011;43(7): 13341359. Available from: doi:10.1249/MSS.0b013e318213fefb

2. Stone $\mathrm{MH}$, Potteiger JA, Pierce KC, Proulx CM, O'bryant HS, Johnson RL, et al. Comparison of the Effects of Three Different Weight-Training Programs on the One Repetition Maximum Squat. The Journal of Strength \& Conditioning Research. 2000;14(3): 332-337.

3. Ullrich B, Pelzer T, Oliveira S, Pfeiffer M. Neuromuscular Responses to ShortTerm Resistance Training With Traditional and Daily Undulating 
Periodization in Adolescent Elite Judoka. The Journal of Strength \& Conditioning Research. [Online] 2016;30(8): 2083-2099. Available from: doi:10.1519/JSC.0000000000001305

4. Harries SK, Lubans DR, Callister R. Systematic review and meta-analysis of linear and undulating periodized resistance training programs on muscular strength. Journal of Strength and Conditioning Research. [Online] 2015;29(4): 1113-1125. Available from: doi:10.1519/JSC.0000000000000712

5. Moraes E, Fleck SJ, Ricardo Dias M, Simão R. Effects on strength, power, and flexibility in adolescents of nonperiodized vs. daily nonlinear periodized weight training. Journal of Strength and Conditioning Research. [Online] 2013;27(12): 3310-3321. Available from: doi:10.1519/JSC.0b013e31828de8c3

6. Miranda F, Simão R, Rhea M, Bunker D, Prestes J, Leite RD, et al. Effects of Linear vs. Daily Undulatory Periodized Resistance Training on Maximal and Submaximal Strength Gains. The Journal of Strength \& Conditioning Research. [Online] 2011;25(7): 1824. Available from: doi:10.1519/JSC.0b013e3181e7ff75

7. Monteiro AG, Aoki MS, Evangelista AL, Alveno DA, Monteiro GA, Piçarro I da $\mathrm{C}$, et al. Nonlinear periodization maximizes strength gains in split resistance training routines. Journal of Strength and Conditioning Research. [Online] 2009;23(4): 1321-1326. Available from: doi:10.1519/JSC.0b013e3181a00f96

8. Prestes J, Frollini AB, de Lima C, Donatto FF, Foschini D, de Cássia Marqueti R, et al. Comparison between linear and daily undulating periodized resistance training to increase strength. Journal of Strength and Conditioning Research. [Online] 2009;23(9): 24372442. Available from: doi:10.1519/JSC.0b013e3181c03548
9. Ayllón FN, Jiménez A, Alvar BA, Peterson MD. Assessing strength and power in resistance training. Journal of Human Sport and Exercise. [Online] 2009;4(2): 100-113. Available from: doi:10.4100/jhse.2009.42.04

10. American College of Sports Medicine. ACSM Guidelines for Exercise Testing and Prescription. 10th ed. Baltimore: Lippincott Williams \& Wilkins; 2017. book.

11. Silva EA da, Lima CZL de, Nassar SE, Oliveira E de, Farias D de A. Periodização ondulatória diária versus ondulatória semanal sobre força máxima e potência de membros inferiores: um estudo experimental longitudinal. Revista de Educação Física / Journal of Physical Education. [Online] 2019;88(4): 999-1010. Available from: doi:10.37310/ref.v88i4.866

12. Rhea MR, Ball SD, Phillips WT, Burkett LN. A comparison of linear and daily undulating periodized programs with equated volume and intensity for strength. Journal of Strength and Conditioning Research. 2002;16(2): 250-255.

13. Mayhew JL, Ball TE, Ward TE, Hart CL, Arnold MD. Relationships of structural dimensions to bench press strength in college males. The Journal of Sports Medicine and Physical Fitness. 1991;31(2): 135-141.

14. Marinho BF, Marins JCB. Teste de força/resistência de membros superiores: análise metodológica e dados normativos. Fisioterapia em Movimento. [Online] PUCPR; 2012;25(1): 219-230. Available from: doi:10.1590/S010351502012000100021

15. Harman EA, Rosenstein MT, Frykman PN, Rosenstein RM. The effects of arms and countermovement on vertical jumping. Medicine and Science in Sports and Exercise. [Online] 1990;22(6): 825833. Available from: doi:10.1249/00005768-19901200000015 
16. Loturco I, Pereira LA, Cal Abad CC, D'Angelo RA, Fernandes V, Kitamura $\mathrm{K}$, et al. Vertical and Horizontal Jump Tests Are Strongly Associated With Competitive Performance in 100-m Dash Events. The Journal of Strength \& Conditioning Research. [Online] 2015;29(7): 1966-1971. Available from: doi:10.1519/JSC.0000000000000849

17. CFST. Canadian Standardized Test of Fitness: Operations Manual. Government of Canada, Fitness and Amateur Sport; 1986. 40 p.

18. Safrit MJ. Complete Guide to Youth Fitness Testing. Human Kinetics; 1995. $145 \mathrm{p}$.

19. Johnson BL, Nelson JK. Practical Measurements for Evaluation in Physical Education. Burgess Pub.; 1986. $504 \mathrm{p}$.

20. Amiri-Khorasani M, Sahebozamani M, Tabrizi KG, Yusof AB. Acute effect of different stretching methods on Illinois agility test in soccer players. Journal of Strength and Conditioning Research. [Online] 2010;24(10): 2698-2704. Available from: doi:10.1519/JSC.0b013e3181bf049c

21. Farias D de A, Willardson JM, Paz GA, Bezerra E de S, Miranda H. Maximal Strength Performance and Muscle Activation for the Bench Press and Triceps Extension Exercises Adopting Dumbbell, Barbell, and Machine Modalities Over Multiple Sets. Journal of Strength and Conditioning Research. [Online] 2017;31(7): 1879-1887. Available from: doi: $10.1519 /$ JSC. 0000000000001651

22. American College of Sports Medicine. American College of Sports Medicine position stand. Progression models in resistance training for healthy adults. Medicine and Science in Sports and Exercise. [Online] 2009;41(3): 687-708. Available from: doi:10.1249/MSS.0b013e3181915670

23. Rhea MR. Determining the magnitude of treatment effects in strength training research through the use of the effect size. Journal of Strength and Conditioning Research. [Online] 2004;18(4): 918-920. Available from: doi:10.1519/14403.1

24. Buford TW, Rossi SJ, Smith DB, Warren AJ. A comparison of periodization models during nine weeks with equated volume and intensity for strength. Journal of Strength and Conditioning Research. [Online] 2007;21(4): 1245-1250. Available from: doi:10.1519/R-20446.1

25. Bartolomei S, Hoffman JR, Stout JR, Zini M, Stefanelli C, Merni F. Comparison of block versus weekly undulating periodization models on endocrine and strength changes in male athletes. Kinesiology. [Online] Kineziološki fakultet; 2016;48.(1.): 7178. Available from: doi: $10.26582 / \mathrm{k} .48 .1 .9$

26. Franchini E, Branco BM, Agostinho MF, Calmet M, Candau R. Influence of Linear and Undulating Strength Periodization on Physical Fitness, Physiological, and Performance Responses to Simulated Judo Matches. The Journal of Strength \& Conditioning Research. [Online] 2015;29(2): 358. Available from: doi:10.1519/JSC.0000000000000460

27. Tammam AH, Hashem EM. Comparison Between Daily and Weekly Undulating Periodized Resistance Training to Increase Muscular Strength for Volleyball Players. Journal of Applied Sports Science. [Online] 2015;5(3). Available from: http://jass.alexu.edu.eg/index.php/JASS/ article/view/89 [Accessed: 27th July 2020]

28. Antretter $M$, Färber S, Immler L, Perktold M, Posch D, Raschner C, et al. The Hatfield-system versus the weekly undulating periodised resistance training in trained males. International Journal of Sports Science \& Coaching. [Online] SAGE Publications; 2018;13(1): 95- 
103. Available from: doi:10.1177/1747954117746457

29. Rodrigues BM, Senna G, Simão R, Scudese E, Silva-Grigoletto MED, Paoli A, et al. Traditional vs daily undulling periodization in strength and local muscle endurance gains on trained men. Journal of Human Sport and Exercise. [Online] 2018;13(2): 401-414. Available from: doi:10.14198/jhse.2018.132.11

30. de Lima C, Boullosa DA, Frollini AB, Donatto FF, Leite RD, Gonelli PRG, et al. Linear and daily undulating resistance training periodizations have differential beneficial effects in young sedentary women. International Journal of Sports Medicine. [Online] 2012;33(9): 723727. Available from: doi:10.1055/s0032-1306324

31. Hopkins WG. Measures of reliability in sports medicine and science. Sports Medicine (Auckland, N.Z.). 2000;30(1): $1-15$.

32. Braith RW, Graves JE, Leggett SH, Pollock ML. Effect of training on the relationship between maximal and submaximal strength. Medicine and Science in Sports and Exercise. 1993;25(1): 132-138.

33. Grgic J, Mikulic P, Podnar H, Pedisic Z. Effects of linear and daily undulating periodized resistance training programs on measures of muscle hypertrophy: a systematic review and meta-analysis. PeerJ. [Online] 2017;5: e3695. Available from: doi:10.7717/peerj.3695

34. Nagano A. Optimal coordination of maximal-effort horizontal and vertical jump motions-a computer simulation study. BioMedical Engineering OnLine. 2007;1(6): 20.

35. Myer G, Brent J, Ford K, Hewett T. A pilot study to determine the effect of trunk and hip focused neuromuscular training on hip and knee isokinetic strength. British Journal of Sports Medicine. [Online] 2008; Available from: doi:10.1136/bjsm.2007.046086 [Accessed: 24th September 2018]

36. O'Bryant HS, Byrd R, Stone MH. Cycle Ergometer Performance and Maximum Leg and Hip Strength Adaptations to Two Different Methods of WeightTraining. The Journal of Strength \& Conditioning Research. 1988;2(2): 27.

37. Peterson MD, Dodd DJ, Alvar BA, Rhea MR, Favre M. Undulation training for development of hierarchical fitness and improved firefighter job performance. Journal of Strength and Conditioning Research. [Online] 2008;22(5): 1683-1695. Available from: doi:10.1519/JSC.0b013e31818215f4

38. Baker D, Wilson G, Carlyon R. Periodization: The Effect on Strength of Manipulating Volume and Intensity. The Journal of Strength \& Conditioning Research. 1994;8(4): 235.

39. Rhea MR, Phillips WT, Burkett LN, Stone WJ, Ball SD, Alvar BA, et al. A comparison of linear and daily undulating periodized programs with equated volume and intensity for local muscular endurance. Journal of Strength and Conditioning Research. 2003;17(1): 82-87.

40. Cyrino ES, Oliveira AR de, Leite JC, Porto DB, Dias RMR, Segantin AQ, et al. Flexibility behavior after 10 weeks of resistance training. Revista Brasileira de Medicina do Esporte. [Online] 2004;10(4): 233-237. Available from: doi:10.1590/S151786922004000400001

41. Rocha CAQC, Moreira MHR, Mesa EIA, Guimarães AC, Dória $\mathrm{CH}$, Dantas EHM. Efeitos de um programa de treinamento concorrente sobre a autonomia funcional em idosas pósmenopáusicas. Revista Brasileira de Ciência e Movimento. [Online] 2015;23(3): 122-134. Available from: doi:10.18511/rbcm.v23i3.5512

42. Yu K-H, Suk M-H, Kang S-W, Shin YA. Effects of combined linear and nonlinear periodic training on physical 
fitness and competition times in finswimmers. Journal of Exercise Rehabilitation. [Online] 2014;10(5): 306-312. Available from: doi:10.12965/jer.140151

43. Christou M, Smilios I, Sotiropoulos K, Volaklis K, Pilianidis T, Tokmakidis SP. Effects of resistance training on the physical capacities of adolescent soccer players. Journal of Strength and Conditioning Research. [Online] 2006;20(4): 783-791. Available from: doi:10.1519/R-17254.1

44. Kell RT, Asmundson GJG. A comparison of two forms of periodized exercise rehabilitation programs in the management of chronic nonspecific lowback pain. Journal of Strength and Conditioning Research. [Online] 2009;23(2): 513-523. Available from: doi:10.1519/JSC.0b013e3181918a6e

45. Rebelatto JR, Calvo JI, Orejuela JR, Portillo JC. Influência de um programa de atividade física de longa duração sobre a força muscular manual e a flexibilidade corporal de mulheres idosas. Brazilian Journal of Physical Therapy. 2006;10(1): 127-132. Available from: doi:10.1590/S141335552006000100017

46. Lima CZL de, Borges EN, Silva EA da, Nassar SE, Oliveira E de, Farias D de A. Efeitos da periodização linear versus ondulatório diária no treinamento de força sobre a flexibilidade. RBPFEX Revista Brasileira de Prescrição e Fisiologia do Exercício. 2018;12(79): 1037-1044.

47. Picanço LM, Silva JJR, Vecchio FBD. Relationship between strength and agility in futsal players. Revista Brasileira de Futsal e Futebol. Instituto Brasileiro de Pesquisa e Ensino em Fisiologia do Exercicio. IBPEFEX; 2012;4(12): 77-87.

48. Maio Alves JMV, Rebelo AN, Abrantes C, Sampaio J. Short-term effects of complex and contrast training in soccer players' vertical jump, sprint, and agility abilities. Journal of Strength and
Conditioning Research. [Online] 2010;24(4): 936-941. Available from: doi:10.1519/JSC.0b013e3181c7c5fd

49. Barbalho M, Gentil P, Raiol R, Del Vecchio FB, Ramirez-Campillo R, Coswig VS. Non-Linear Resistance Training Program Induced Power and Strength but Not Linear Sprint Velocity and Agility Gains in Young Soccer Players. Sports. [Online] 2018;6(2). Available from: doi:10.3390/sports6020043 [Accessed: 28th September 2018]

50. Keiner M, Sander A, Wirth K, Schmidtbleicher D. Long-term strength training effects on change-of-direction sprint performance. Journal of Strength and Conditioning Research. [Online] 2014;28(1): 223-231. Available from: doi:10.1519/JSC.0b013e318295644b 